\title{
Efeito do Treino de Composição (Cópia) na Aprendizagem do Conceito de Proporção
}

\author{
Antonio Carlos Godinho Santos ${ }^{1}$ \\ Lorismario Ernesto Simonassi \\ Camila Fernandes Rodrigues \\ Maira Ribeiro Magri \\ Pontifícia Universidade Católica de Goiás
}

\begin{abstract}
RESUMO - Este experimento investigou o efeito do treino de relações condicionais entre estímulos fracionários na forma de figuras e numéricos, com e sem treino de composição, sobre a aprendizagem do conceito de proporção. Avaliou-se a formação de classes de equivalência, sua expansão e generalização, e a resolução de problemas, com lápis e papel, com estímulos fracionários. Participaram 20 alunos do sexto ano do Ensino Fundamental. Os grupos GEQ e GEQTC passaram por treinos e testes de relações condicionais, mas este último foi exposto, adicionalmente, ao treino de composição de frações antes dos treinos das relações condicionais; dois grupos controle fizeram apenas as avaliações inicial e final. Os resultados indicaram a formação de classes de equivalência, mas não evidenciaram efeito do treino de composição.
\end{abstract}

Palavras-chave: equivalência de estímulos, aprendizagem de frações, conceito de proporção, treino de composição

\section{The effect of Composition Training (Copy) on Proportion-Concept Learning}

\begin{abstract}
This experiment investigated the effect of training conditional relations between pictorial and numerical fractional stimuli, with and without composition training, upon the learning of the proportion concept. It was evaluated the formation of equivalence classes, expansion of classes formed, generalization to new situations, and pencil and paper problem solving with fractional stimuli. The participants were 20 students of the sixth grade of Elementary School. The QEG and TCQEC groups were exposed to training and testing of conditional relations, but the last group was exposed, additionally, to a fractional composition training before the conditional relations training; two control groups were exposed only to the initial and final tests. The results indicated the formation of equivalence classes, but did not show effects of the composition training.
\end{abstract}

Keywords: stimulus equivalence, fractions learning, concept of proportion, composition training

Uma fração é um número que representa uma parte de um todo (de um inteiro) e é apresentada na forma matemática como $\mathbf{a} / \mathbf{b}$, sendo que $\mathbf{b}$ deve ser diferente de zero. Nesse caso, o símbolo a é chamado de numerador e b de denominador. Frações podem ser expressas de forma absoluta (literal) ou relativa. Por exemplo, um retângulo dividido em três partes iguais e tendo uma delas marcada pode ser representado de forma absoluta pela fração $1 / 3$, uma vez que o numerador corresponde à parte do inteiro que foi destacada e o denominador ao número de partes em que o inteiro foi dividido. Por outro lado, pode ser representado de forma relativa pela fração $2 / 6$, mesmo na ausência de correspondência entre as partes da figura e o numerador e denominador da fração (Carraher \& Schliemann, 1992). Quando notações fracionárias diferentes representam uma mesma quantidade (e.g., 1/3 e 2/6) são denominadas como frações equivalentes.

Frações equivalentes mantêm uma relação de proporcionalidade entre elas. Diz-se que duas frações são proporcionais quando o resultado da multiplicação entre o

1 Endereço para correspondência: Rua Serra Grande,Qd 11, Lt 07, Cj Sonho Verde, Goiânia, GO, Brasil. CEP: 74.730-570. E-mail: acgodinhos@yahoo.com.br numerador da fração 1 e o denominador da fração 2 é igual ao resultado da multiplicação entre o numerador da fração 2 e o denominador da fração 1 . Por exemplo, $1 / 3$ e 2/6 são frações proporcionais, porque 1x6 é igual a 2x3 (Carraher, Carraher, \& Schliemann, 1986). Estudos mostram que alunos do sexto ano do Ensino Fundamental apresentam dificuldades para resolver problemas fracionários que exigem o conceito de proporção (e.g., Carraher \& Shliemann, 1992; Santos, 1999). Em geral, alunos resolvem problemas fracionários quando os problemas apresentam frações absolutas, mas erram quando apresentam frações relativas.

A literatura sobre equivalência de estímulos evidencia que o treino de relações condicionais, segundo o paradigma proposto por Sidman e Taiby (1982), pode ser uma forma alternativa e eficiente para o ensino de conceitos matemáticos mais básicos, como os de número (Carmo, 2000) e de quantidade (Green, 2010), e de conceitos mais complexos que envolvem relações trigonométricas (Ninness et al., 2006; Ninness et al., 2009). Existem também sugestões quanto à aplicação do paradigma de equivalência de estímulos na aprendizagem de relações entre expressões numéricas fracionárias e decimais (Lynch \& Cuvo, 1995) e do conceito de proporção e de equivalência entre frações (Santos, Souza, \& Bay, 1997; Santos, Cameschi, \& Hanna, 2009). 
Em geral, nos procedimentos desses três últimos estudos foram ensinadas relações condicionais, segundo o paradigma de relação de equivalência, entre estímulos fracionários pictóricos (figuras) e numéricos e entre estímulos numéricos expressos de forma absoluta e relativa. Os resultados mostraram que os participantes aprenderam as relações condicionais ensinadas diretamente e demonstraram relações derivadas de simetria, transitividade e equivalência. Contudo, os desempenhos observados em testes de generalização (com novas frações) e, em especial, nos testes de expansão das classes de equivalência, foram inconsistentes entre os participantes.

Pesquisas têm demonstrado a eficiência do paradigma de equivalência de estímulos no ensino de leitura e escrita (Albuquerque \& Melo, 2005; de Rose, Souza, Rossito, \& de Rose, 1989; Sidman, 1971; Sidman \& Cresson,1973). Algumas incluíram, no delineamento, o procedimento de escrever palavras, chamado de treino de composição (de Rose, de Souza, \& Hanna, 1996; de Souza et al.,2009; Hübner, Gomes, \& McIlvane, 2009). Diferentes variáveis foram manipuladas nesses estudos e, de forma geral, os resultados mostraram efeito desse treino sobre a aprendizagem de palavras treinadas e a generalização para novas palavras. Uma possível explicação para esses resultados é que o treino viabiliza o controle de unidades menores (letras e sílabas) sobre o comportamento, a partir de relações formais ou funcionais entre os estímulos e os produtos das respostas (de Rose, 2005; Skinner, 1978).

Considerando o comportamento conceitual matemático como um caso especialmente complexo de relações simbólicas do comportamento verbal, em que as respostas estão sob controle de propriedades ou relações quantitativas (de Rose, 2010), é possível, como ocorre na leitura e escrita, que relações de controle por unidades de diferentes tamanhos controlem o comportamento matemático, o que torna relevante investigar se o treino de composição com estímulos fracionários facilita a aprendizagem do conceito de proporção. Assim, o presente experimento teve como objetivo investigar o efeito do treino de relações condicionais entre estímulos fracionários na forma de figuras e numéricos e entre estímulos numéricos e numéricos, com e sem treino de composição (cópia) sobre: (1) a formação de classes de equivalência com estímulos fracionários; (2) a expansão das classes de equivalência formadas e a generalização para novas situações; e (3) a resolução de problemas fracionários com lápis e papel.

\section{Método}

\section{Participantes}

Participaram do estudo 20 alunos do final do sexto ano do Ensino Fundamental de uma escola pública de Goiânia, com idades entre 11 e 13 anos, de ambos os sexos, que tiverem escores máximos iguais a $30 \%$ de acertos em uma situação de teste denominada como avaliação inicial (descrita a seguir) e cujos responsáveis autorizaram a participação por meio do Termo de Autorização Livre e Esclarecido, conforme orientação do Comitê de Ética e Pesquisa (Processo 06839912.0.0000.037).

\section{Local}

As sessões experimentais ocorreram em uma sala da escola, com iluminação natural e artificial, ventilação natural, mesas medindo $0,60 \mathrm{~cm} \times 0,80 \mathrm{~cm}$, lousa e carteiras escolares.

\section{Estímulos}

Estímulos visuais -frações numéricas e figuras em forma de barra que representavam as frações - foram organizados em quatro conjuntos de três membros (Tabela 1)

Foram utilizados, ainda, os numerais 1,2 e 3, as figuras de uma bola, uma seta e de um sol e seus respectivos nomes, na condição de treino da tarefa (descrita no procedimento).

\section{Material}

Foi utilizada uma pasta do tipo classificador, com folhas ofício do tamanho A4, para apresentar os estímulos de cada condição experimental (Pasta 1). Cada folha apresentava quatro quadrados de $5 \mathrm{~cm} \times 5 \mathrm{~cm}$, sendo um localizado na parte central superior e os três outros, na parte inferior.

Uma pasta contendo figuras representativas das frações numéricas dos conjuntos B, C e D (ver Tabela 1), em forma de barra, medindo $15 \mathrm{~cm} \mathrm{x} 4 \mathrm{~cm}$, também foi utilizada (Pasta 2). Cada figura foi apresentada em uma folha e

Tabela 1. Estímulos apresentados nas condições de treinos e testes das relações condicionais

\begin{tabular}{|c|c|c|c|c|c|c|c|}
\hline \multicolumn{4}{|c|}{ Treinos e Testes de Equivalência } & \multicolumn{4}{|c|}{ Testes de Generalização } \\
\hline Conjunto & 1 & 2 & 3 & Conjunto & 1 & 2 & 3 \\
\hline A & & 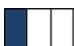 & 7 & $\mathrm{E}$ & $1 / 2$ & $1 / 6$ & $1 / 7$ \\
\hline $\mathrm{B}$ & $1 / 3$ & $1 / 4$ & $1 / 5$ & $\mathrm{~F}$ & $2 / 4$ & $2 / 12$ & $2 / 14$ \\
\hline $\mathrm{C}$ & $2 / 6$ & $2 / 8$ & $2 / 10$ & G & $3 / 6$ & $3 / 18$ & $3 / 21$ \\
\hline $\mathrm{D}$ & $3 / 9$ & $3 / 12$ & $3 / 15$ & - & - & - & - \\
\hline
\end{tabular}


continha partes azuis e brancas, sendo as azuis referentes aos numeradores e as brancas aos denominadores das frações.

Finalmente, foi utilizado um tabuleiro de madeira com $20 \mathrm{~cm}$ de largura, $30 \mathrm{~cm}$ de comprimento, $1,5 \mathrm{~cm}$ de altura e $1 \mathrm{~cm}$ de profundidade. Esse tabuleiro foi dividido em quatro espaços, separados por divisórias de $1 \mathrm{~cm}$ de espessura, nos quais foram encaixados tocos de madeira de formato retangular. O primeiro espaço foi usado para encaixar um toco de cor branca com $19 \mathrm{~cm}$ de comprimento, $5 \mathrm{~cm}$ de largura e $1,5 \mathrm{~cm}$ de altura. Os três espaços restantes destinaram-se ao encaixe de tocos menores cujos comprimentos variaram de acordo com o número de peças necessárias para representar as frações $1 / 3,2 / 6,3 / 9$, no segundo, terceiro e quarto espaços, respectivamente. $\mathrm{O}$ mesmo critério foi adotado para os tocos referentes às frações $1 / 4,2 / 8$ e 3/12 e 1/5, 2/10 e 3/15. O comprimento de todas as peças menores foi igual ao do toco branco inteiro. As peças que representaram os numeradores eram azuis e as que representaram os denominadores, brancas.

\section{Procedimento}

Foram organizados quatro grupos de cinco participantes. Um grupo, denominado de Grupo Equivalência (GEQ), passou por treinos (AB, BC e AD) e testes (BA, CB e DA) de relações condicionais, de acordo com o paradigma de equivalência proposto por Sidman e Tailby (1982). Outro grupo, nomeado de Grupo Equivalência com Treino de Composição (GEQTC), foi exposto aos mesmos treinos e testes, mas recebeu treino de composição antes das condições de treino, após o teste de transitividade/ equivalência e após o teste de expansão da rede. As avaliações inicial e final, realizada antes e após os treinos e testes, respectivamente, foram realizadas ao mesmo tempo por um par de participantes, um participante dos grupos GEQ ou GEQTC e um participante do grupo controle, possibilitando que o tempo entre as duas avaliações fosse o mesmo para cada par de participante. Dessa forma, havia dois grupos controle, um para cada grupo experimental. A Tabela 2 descreve as condições e procedimentos adotados para cada grupo.

Tabela 2. Organização das condições experimentais de treinos e testes

\begin{tabular}{|c|c|c|c|c|}
\hline \multirow{2}{*}{ Condição } & \multirow{2}{*}{ Relações Apresentadas } & \multicolumn{3}{|c|}{ Grupos } \\
\hline & & GEQ & GEQTC & Controles \\
\hline Avaliação Inicial & Ver no texto & $\operatorname{Sim}$ & Sim & Sim \\
\hline Teste de conhecimentos básicos & Ver no texto & $\operatorname{Sim}$ & Sim & Não \\
\hline Treino da tarefa & Ver no Texto & Sim & Sim & Não \\
\hline Treino AB & $\mathrm{A} 1 \mathrm{~B} 1, \mathrm{~A} 2 \mathrm{~B} 2$ e $\mathrm{A} 3 \mathrm{~B} 3$ & Sim & Sim & Não \\
\hline Treino de Composição 1 & Frações $1 / 3,1 / 4$ e $1 / 5$ & Não & Sim & Não \\
\hline Treino BC & $\mathrm{B} 1 \mathrm{C} 1, \mathrm{~B} 2 \mathrm{C} 2$ е $\mathrm{B} 3 \mathrm{C} 3$ & Sim & Sim & Não \\
\hline Treino de Composição 2 & Frações $2 / 6,2 / 8$ e $2 / 10$ & Não & Sim & Não \\
\hline Treino Misto & $\mathrm{A} 1 \mathrm{~B} 1, \mathrm{~A} 2 \mathrm{~B} 2, \mathrm{~A} 3 \mathrm{~B} 3, \mathrm{~B} 1 \mathrm{C} 1, \mathrm{~B} 2 \mathrm{C} 2$ e B3C3 & Sim & Sim & Não \\
\hline Teste de Simetria & $\mathrm{B} 1 \mathrm{~A} 1, \mathrm{~B} 2 \mathrm{~A} 2, \mathrm{~B} 3 \mathrm{~A} 3, \mathrm{C} 1 \mathrm{~B} 1, \mathrm{C} 2 \mathrm{~B} 2$ e $\mathrm{C} 3 \mathrm{~B} 3$ & Sim & Sim & Não \\
\hline Treino de simetria & $\mathrm{B} 1 \mathrm{~A} 1, \mathrm{~B} 2 \mathrm{~A} 2, \mathrm{~B} 3 \mathrm{~A} 3, \mathrm{C} 1 \mathrm{~B} 1, \mathrm{C} 2 \mathrm{~B} 2$ e $\mathrm{C} 3 \mathrm{~B} 3$ & Sim & Sim & Não \\
\hline Teste de Transitividade e equivalência & $\mathrm{A} 1 \mathrm{C} 1, \mathrm{~A} 2 \mathrm{C} 2, \mathrm{~A} 3 \mathrm{C} 3, \mathrm{C} 1 \mathrm{~A} 1, \mathrm{C} 2 \mathrm{~A} 2$ e $\mathrm{C} 3 \mathrm{~A} 3$ & Sim & Sim & Não \\
\hline Treino de Composição 3 & Frações $1 / 3$ e $2 / 6 ; 1 / 4$ e $2 / 8$ e $1 / 5$ e $2 / 10$ & Não & Sim & Não \\
\hline Treino de Transitividade e equivalência & $\mathrm{A} 1 \mathrm{C} 1, \mathrm{~A} 2 \mathrm{C} 2, \mathrm{~A} 3 \mathrm{C} 3, \mathrm{C} 1 \mathrm{~A} 1, \mathrm{C} 2 \mathrm{~A} 2$ e $\mathrm{C} 3 \mathrm{~A} 3$ & Sim & Sim & Não \\
\hline Teste de Generalização 1 & Ver no texto & Sim & Sim & Não \\
\hline Treino de AD & $\mathrm{A} 1 \mathrm{D} 1, \mathrm{~A} 2 \mathrm{D} 2 \mathrm{e} \mathrm{A} 3 \mathrm{D} 3$ & Sim & Sim & Não \\
\hline Treino de Composição 4 & Frações $3 / 9,3 / 12$ e $3 / 15$ & Não & Sim & Não \\
\hline Teste de Expansão das classes D-(ABC) & $\begin{array}{l}\text { D1A1, D2A2, D3A3, D1B1, D2B2, D3B3, B1D1, } \\
\text { B2D2, B3D3, C1D1, C2D2, C3D3, D1C1, D2C2 e } \\
\text { D3C3 }\end{array}$ & Sim & Sim & Não \\
\hline Treino de Expansão da rede D-(ABC) & Idem ao teste de Expansão da rede D-(ABC) & Sim & Sim & Não \\
\hline Treino de Composição 5 & $\begin{array}{l}\text { Frações } 1 / 3,2 / 6 \text { e } 3 / 9 ; 1 / 4,2 / 8 \text { e } 2 / 10 ; 1 / 5,2 / 10 \text { e } \\
3 / 15\end{array}$ & Não & Sim & Não \\
\hline Teste de Generalização 2 & Ver no texto & Sim & Sim & Não \\
\hline Avaliação Final & Ver no texto & Sim & Sim & Sim \\
\hline
\end{tabular}

Nota. GEQ refere-se ao grupo Equivalência e GEQTC, ao grupo Equivalência mais Treino de Composição. 
Grupo Equivalência (GEQ). Os participantes foram expostos, em ordem, às seguintes condições de treinos e testes de relações condicionais: avaliação inicial, treino da tarefa, treino $\mathrm{AB}$, treino $\mathrm{BC}$, treino $\mathrm{AB} / \mathrm{BC}$, teste de simetria $(\mathrm{BA} / \mathrm{CB})$, teste de transitividade/equivalência ( $\mathrm{AC} / \mathrm{CA})$, teste de generalização 1 , treino $\mathrm{AD}$, teste $\mathrm{D}-(\mathrm{ABC})$, teste de generalização 2 e avaliação final.

Avaliação inicial. Essa avaliação consistiu na aplicação coletiva de uma prova contendo 30 problemas fracionários, sendo que 10 apresentaram relações absolutas e 20, relações relativas [detalhes da prova podem ser encontrados em Santos (1999)].

Treino da tarefa. Todos os participantes receberam, inicialmente, treino na tarefa de pareamento com o modelo com seis estímulos não fracionários. Foram ensinadas seis relações entre quantidades de figuras geométricas e numerais, entre numerais e palavras e quantidades de figuras geométricas e palavras. Por exemplo, uma bolinha como modelo e os numerais 1,2 e 3 como comparação. $O$ treino compreendeu dois blocos com nove tentativas. Os estímulos foram apresentados na Pasta 1 descritas no item "Material". Os estímulos do modelo apareceram no quadrado superior e os três estímulos de comparação, nos inferiores. Os acertos foram consequenciados com a vocalização "certo" e os erros, com "errado", sendo que, nesse caso, um procedimento de correção (repetição da tentativa) vigorou até o acerto ocorrer. As instruções orais foram:

Sua tarefa é simples. Você deverá, em primeiro lugar, tocar no quadrado da parte de cima da folha. Em seguida, você deverá tocar uma das figuras laterais. Eu the direi se sua escolha foi certa ou errada. Tente acertar o máximo possível. Você entendeu?

Treinos $A B, B C$ e $A D$. Cada condição de treino foi precedida por um pré-teste, ou seja, por um bloco de nove tentativas com as relações que seriam posteriormente ensinadas. Nesse pré-teste não havia nenhuma consequência programada para as respostas de escolha. Apenas quem teve menos que $100 \%$ de acertos no pré-teste foi exposto à condição de treino. Após o treino, o bloco de nove tentativas foi novamente aplicado e denominado de pós-teste. As seguintes instruções orais antecederam os pré- e pós-testes:

Sua tarefa continua sendo simples. Primeiramente, toque o quadrado que está na parte de cima da folha e em seguida toque uma das frações abaixo. Não será dito a você se sua escolha foi certa ou errada. Portanto, você poderá estar errando ou acertando em suas escolhas. Tente acertar o máximo que você puder. Entendeu?.

Nas condições dos treinos $\mathrm{AB}$ e $\mathrm{AD}$ ensinaram-se relações entre estímulos na forma de figuras como modelo (conjunto A) e frações numéricas (conjuntos B e D) como comparações. No treino BC, tanto os modelos (B) quanto as comparações $(\mathrm{C})$ foram frações numéricas. A definição alfanumérica (A1B1, B1C1, A1D1, etc) determinou quais relações foram consideradas certas ou erradas (Tabela 1). A configuração da apresentação dos estímulos de modelo e comparação foi igual à do treino da tarefa, sendo que as comparações corretas apareceram em posições diferentes a cada tentativa. Cada relação foi apresentada três vezes, formando um bloco de treino com nove tentativas. Cada condição de treino apresentou, alternadamente, dois blocos que diferiram apenas quanto à ordem de apresentação das tentativas. As seguintes instruções orais foram fornecidas:

\section{Sua tarefa continua sendo simples. Tudo o que você tem que fazer é tocar na figura da parte de cima da folha e, em seguida, tocar uma das frações abaixo. No entanto, agora será dito a você se sua escolha foi certa ou errada. Tente acertar o máximo possivel. Você entendeu?.}

No treino $\mathrm{AB} / \mathrm{BC}$ foram aplicados dois blocos com 12 tentativas, sendo seis relações $\mathrm{AB}$ e seis, $\mathrm{BC}$. Os blocos diferiram apenas quanto a ordem de apresentação das tentativas e apenas três delas em cada bloco foram consequenciadas. O critério adotado para o encerramento de todas as condições de treino foi o acerto de $100 \%$ das tentativas a partir da exposição ao segundo bloco. As instruções orais foram:

A sua tarefa é igual à anterior. Tudo que você tem que fazer é tocar na figura da parte de cima da folha e em seguida tocar uma das frações abaixo. Apenas algumas vezes será dito a você se sua escolha foi certa ou errada. Quando nada for dito você pode ter acertado ou errado. Entendeu?.

Condições de testes. Os testes avaliaram a emergência das relações $\mathrm{BA} / \mathrm{CB}$ (simetria) e AC/CA (transitividade/ equivalência), expansão D-(ABC) e generalização (Tabela 1). Cada teste foi programado separadamente e consistiu na apresentação alternada de dois blocos de 12 tentativas cada. No teste de simetria, três tentativas envolveram a relação $\mathrm{BA}$, três, a relação $\mathrm{CB}$, e seis, a relação $\mathrm{AB}$ e $\mathrm{BC}$ (três de cada relação). De forma semelhante, no teste $\mathrm{AC} / \mathrm{CA}$, houve três tentativas com a relação $\mathrm{AC}$, três com a relação $\mathrm{CA}$ e seis com as relações $\mathrm{AB}$ e $\mathrm{BC}$. Os dois blocos dessas duas condições diferiram apenas quanto à ordem de apresentação das tentativas. Apenas quatro, das seis tentativas de treino de cada bloco, foram consequenciadas. O teste $\mathrm{D}-(\mathrm{ABC})$ avaliou a ocorrência das seguintes relações: DA, DB, BD, DC e $\mathrm{CD}$. Apresentaram-se alternadamente dois blocos formados por três tentativas de cada relação (variando a posição da comparação correta) e mais cinco tentativas de linha de base, sendo estas as únicas consequenciadas. A ordem das tentativas variou em cada bloco.

Os testes se encerraram com base em um de dois critérios: (a) $100 \%$ de acerto a partir do segundo bloco de tentativas, ou (b) em caso de erros no segundo bloco, o participante era exposto a blocos adicionais de tentativas até demonstrar $100 \%$ de acerto; porém, se a porcentagem de acerto diminuísse de um bloco para outro, o teste era suspenso e as relações eram treinadas segundo critérios dos treinos anteriores. Ou seja, os treinos consistiram na aplicação dos blocos de testes com todas as tentativas consequenciadas. Nesta condição, as instruções foram as mesmas dos treinos AB/BC.

$\mathrm{O}$ teste de generalização 1 avaliou as relações E1F1, E2F2, E3F3, F1E1, F2E2 e F3E3. Apresentou-se um bloco com três tentativas de cada relação (variando-se a posição 
da comparação correta) e mais quatro tentativas de linha de base que foram consequenciadas. No teste de generalização 2 foram avaliadas as relações E1G1, E2G2, E3G3, G1E1, G2E2, G3E3, F1G1, F2G2, F3G3. Apresentou-se um bloco com três tentativas de cada relação (variando-se a posição da comparação correta) e seis tentativas de linha de base que foram consequenciadas. As instruções foram idênticas às da condição de treino $\mathrm{AB} / \mathrm{BC}$.

Após o teste de generalização 2, os participantes resolveram os mesmos problemas aplicados na avaliação inicial que agora foi denominada de avaliação final.

Grupo Equivalência mais Treino de Composição (GEQTC). O grupo GEQTC passou pelas mesmas condições programadas para o grupo GEQ. Adicionalmente, foi exposto a treinos de composição, conforme descrição a seguir.

Treinos de composição. Os treinos de composição ocorreram antes das condições de treino AB, BC e AD, após o teste ou treino de transitividade/equivalência, e após o teste ou treino de expansão. Durante os treinos de composição, o tabuleiro ficou do lado direito da mesa e os toquinhos, do lado esquerdo. Usando os tocos, os participantes construíram, no tabuleiro, cópias das figuras-modelo representativas de frações que ficaram à mostra durante toda a tarefa. As figurasmodelo eram retângulos divididos em partes iguais, com partes azuis e brancas (Conjunto A). As azuis corresponderam aos numeradores e as brancas, aos denominadores de representações fracionárias numéricas (por exemplo, um retângulo dividido em três partes iguais, sendo uma azul e duas brancas correspondeu à fração $1 / 3$ ).

O toco inteiro e branco esteve sempre no primeiro espaço do tabuleiro. Antes de cada composição, o experimentador dizia o nome da fração que representava a figura-modelo e o participante devia repeti-lo em voz alta (ecóico). Os participantes tinham 5 min para fazer cada composição. Caso não fizesse a tarefa ou a fizesse de forma incorreta, o experimentador mostrava como deveria ser feita e passava para uma nova tentativa.

Foram programados cinco treinos de composição ao longo do experimento. No primeiro, após o pré-teste $\mathrm{AB}$, ficaram sobre a mesa, expostos de forma aleatória, os toquinhos referentes às frações $1 / 3,1 / 4$ e $1 / 5$ e os participantes compuseram, nessa ordem e no segundo espaço do tabuleiro, um objeto semelhante à figura-modelo (apresentada na pasta) referente a cada uma dessas frações. Após cada composição, os toquinhos eram retirados do tabuleiro. Em seguida, ocorreram as recomposições de todas as frações, sendo a composição da fração $1 / 3$, no segundo espaço do tabuleiro, e as das frações $1 / 4$ e $1 / 5$, no terceiro e quarto espaços, respectivamente. Todas as composições permaneceram nos espaços utilizados para esse fim. Nessa tarefa, os toquinhos da fração 2/6 estiveram disponíveis sobre a mesa. Em seguida, todos os toquinhos foram retirados do tabuleiro. O segundo treino de composição ocorreu após o pré-teste $\mathrm{BC}$ e nele foram utilizados toquinhos e figurasmodelo das frações $2 / 6,2 / 8$ e $2 / 10$. O procedimento, critérios e instruções foram os mesmos utilizados para o treino de composição realizado após o pré-teste AB. Durante essa condição também estiveram sobre a mesa os toquinhos referentes à fração $1 / 4$.
O terceiro treino de composição ocorreu após o teste de transitividade/equivalência e nele foram utilizados toquinhos e figuras-modelo das frações 2/6, 2/8, 2/10 (Conjunto B) e 3/9, 3/12 e 3/15 (Conjunto C). Esse treino ocorreu em três fases. Na primeira, os participantes compuseram a fração $1 / 3$ no segundo espaço do tabuleiro, a qual permaneceu no local. Posteriormente, compuseram a fração 2/6 no terceiro espaço do tabuleiro. Em seguida, todos os toquinhos foram retirados e o mesmo procedimento foi realizado em relação às frações $1 / 4$ e $2 / 8$ (segunda fase), e por último, com as frações $1 / 5$ e 2/10 (terceira fase). Nesse treino, os toquinhos referentes a todas as frações ficaram dispostos de forma aleatória sobre a mesa em todas as fases. Os critérios e instruções foram idênticos aos do primeiro treino de composição. O quarto treino de composição ocorreu após o pré-teste $\mathrm{AD}$ e obedeceu aos mesmos critérios e instruções do terceiro treino. Contudo, a tarefa foi realizada com as frações 3/9, 3/12 e $3 / 15$. O quinto treino de composição aconteceu antes do teste de expansão e foi divido em três fases. Na primeira, os participantes compuseram a fração $1 / 3$ no segundo espaço do tabuleiro. Depois, compuseram a fração $2 / 6$ e 3/9 nos terceiro e quarto espaços, respectivamente. Todas as composições permaneceram em seus respectivos espaços no tabuleiro. Em seguida, todos os toquinhos foram retirados e o mesmo procedimento foi realizado com as frações $1 / 4,2 / 8$ e $3 / 12$ (segunda fase) e, por fim, com as frações $1 / 5,2 / 10$ e $3 / 15$ (terceira fase). Os toquinhos de todas as frações ficaram dispostos aleatoriamente sobre a mesa. Nas fases um e três foram adicionados os toquinhos referentes à fração $2 / 8$ e na fase dois, os toquinhos da fração $2 / 6$. A instrução para a tarefa de composição dos objetos foi:

Você tem à sua frente vários toquinhos brancos e azuis. Tem também um tabuleiro (apontar). Sua tarefa será olhar para a figura que eu lhe mostrarei nesta pasta (apontar) e, usando os toquinhos, fazer um objeto igual à figura que você verá no espaço abaixo do toco inteiro que eu lhe mostrar (indicar). Você poderá ver a figura o tempo todo. O objeto que você vai fazer, ou seja, a cópia que você fará, deverá ficar com o mesmo tamanho do toco inteiro que está na parte de cima do tabuleiro e conter o mesmo número de partes da figura, sendo que todos os toquinhos que você usar deverão ter o mesmo tamanho. Você terá cinco minutos para fazer essa tarefa.

Quando o participante não fez a tarefa ou a fez de forma incorreta após $5 \mathrm{~min}$, o experimentador descreveu, passo a passo, a forma correta de construir o objeto a partir das seguintes instruções orais:

Seu tempo acabou. Vou lhe mostrar como fazer a tarefa. Primeiro, conte quantos toquinhos são de cor branca e quantos são de cor azul na figura que eu lhe mostrei [se o participante errasse ou não soubesse a resposta, o experimentador contava junto com ele]. Agora, ache entre todos os toquinhos que estão sobre a mesa, aqueles que têm tamanhos iguais aos da figura e coloque-os em separado [se, após 3 min, o participante não identificasse os toquinhos, o experimentador mostrava ao participante quais eram os toquinhos corretos]. Agora, coloque-os nesse espaço do tabuleiro [experimentador indicava o espaço] na mesma ordem da figura [se o participante não soubesse, 
o experimentador indicava qual era o toco e o local que ele deveria ser colocado]. Faça isso com os demais toquinhos.

Pronto, agora você têm um objeto igual ao da figura da pasta.

Grupos Controle. Os participantes dos grupos de controle foram expostos apenas às avaliações inicial e final, seguindo as mesmas instruções e critérios aos quais o participantes dos grupos GEQ e GEQTC foram submetidos.

\section{Resultados}

A Tabela 3 apresenta as porcentagens (individuais e médias) de acerto nas frações, expressas de forma absoluta e relativa nas avaliações inicial e final, para os participantes dos grupos GEQ, GEQTC e seus respectivos grupos controle.

Em todas as avaliações, as porcentagens médias de acertos de todos os grupos foram maiores quando as frações foram expressas de forma absoluta. Para os grupos experimentais observou-se aumento na porcentagem média de acerto da avaliação inicial para a final com frações absolutas. Para o grupo GEQ, a média passou de $74 \%$ para $94 \%$, e para o GEQTC, o aumento foi de $58 \%$ para $80 \%$. Apesar dos resultados mostrarem ganhos de $27 \%$ no GEQ e $38 \%$ no GEQTC, o teste $t$ mostrou que as diferenças entre essas médias não foram significativas ( $p=0,06$ no GEQ e $p=0,09$ no GEQTC). Para os grupos controle, por outro lado, observaram-se decréscimos nas porcentagens médias de acerto nas avaliações finais com frações absolutas. No grupo controle-GEQ, diminui de $72 \%$ para $60 \%$, e no controle-GEQTC, de $84 \%$ para $80 \%$. Apesar dos resultados mostrarem reduções de $16 \%$ no controle-GEQ e $4,8 \%$ no controle-GEQTC, o teste $t$ mostrou que não houve diferença significativa entre as médias $(p=0,28$ para o controle-GEQ e 0,42 para o controle-GEQTC).

Quando as frações foram expressas de forma relativa, houve ganhos na porcentagem média de acerto nos grupos experimentais e nos de controle. Nos grupos experimentais houve aumento de $17 \%$ para $28 \%$ para o GEQ e de $14 \%$ para $48 \%$ para o GEQTC. Apesar dos resultados mostrarem aumento de $65 \%$ no GEQ e $243 \%$ no GEQTC, o teste $\mathrm{t}$ mostrou que as diferenças entre essas médias não foram significativas ( $p=0,22$ para o GEQ e $p=0,06$ para o GEQTC). No grupo controle-GEQ houve aumento de $13 \%$ para $18 \%$, e no controle-GEQTC, de $17 \%$ para $24 \%$. Apesar dos resultados mostrarem aumentos de $38 \%$ para o controle-GEQ e $41 \%$ para o controle-GEQTC, o teste $t$ mostrou que essas diferenças não foram significativas $(p=0,16$ para o controleGEQ e $p=0,18$ para o controle-GEQTC).

$\mathrm{O}$ teste $t$ mostrou não haver diferença significativa entre os grupos GEQ e seu controle quando as frações foram expressas de forma relativa $(p=0,26)$, mas quando as frações

Tabela 3. Porcentagem (individual e média) de acertos nas frações expressas de forma absoluta e relativa

\begin{tabular}{|c|c|c|c|c|c|c|c|c|c|}
\hline \multicolumn{10}{|c|}{ GEQ } \\
\hline \multicolumn{5}{|c|}{ Grupo Experimental } & \multicolumn{5}{|c|}{ Grupo Controle } \\
\hline & \multicolumn{2}{|c|}{ Absolutas } & \multicolumn{2}{|c|}{ Relativas } & & \multicolumn{2}{|c|}{ Absolutas } & \multicolumn{2}{|c|}{ Relativas } \\
\hline Participante & A.I & A.F & A.I & A.F & Participante & A.I & A.F & A.I & A.F \\
\hline $\mathrm{P} 1$ & 30 & 100 & 25 & 0 & P11 & 90 & 90 & 15 & 20 \\
\hline $\mathrm{P} 2$ & 90 & 80 & 20 & 10 & $\mathrm{P} 12$ & 60 & 50 & 10 & 20 \\
\hline P3 & 90 & 100 & 10 & 25 & P13 & 40 & 30 & 15 & 0 \\
\hline P4 & 80 & 90 & 10 & 25 & $\mathrm{P} 14$ & 100 & 80 & 10 & 25 \\
\hline P5 & 80 & 100 & 20 & 80 & $\mathrm{P} 15$ & 70 & 50 & 15 & 25 \\
\hline -11 & 74 & 94 & 17 & 28 & एा & 72 & 60 & 13 & 18 \\
\hline$\sigma$ & 25,1 & 8,9 & 6,7 & 30,9 & & 23,9 & 24,5 & 2,7 & 10,4 \\
\hline \multicolumn{10}{|c|}{ GEQTC } \\
\hline \multicolumn{5}{|c|}{ Grupo Experimental } & \multicolumn{5}{|c|}{ Grupo Controle } \\
\hline & \multicolumn{2}{|c|}{ Absolutas } & \multicolumn{2}{|c|}{ Relativas } & & \multicolumn{2}{|c|}{ Absolutas } & \multicolumn{2}{|c|}{ Relativas } \\
\hline Participante & A.I & A.F & A.I & A.F & Participante & A.I & A.F & A.I & A.F \\
\hline P6 & 60 & 70 & 5 & 5 & P16 & 100 & 80 & 20 & 50 \\
\hline $\mathrm{P} 7$ & 70 & 100 & 15 & 95 & P17 & 100 & 90 & 15 & 25 \\
\hline P8 & 60 & 90 & 0 & 25 & P18 & 20 & 30 & 15 & 10 \\
\hline P9 & 80 & 100 & 30 & 95 & P19 & 100 & 100 & 10 & 15 \\
\hline P10 & 20 & 40 & 20 & 20 & $\mathrm{P} 20$ & 100 & 100 & 25 & 20 \\
\hline 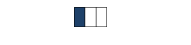 & 58 & 80 & 14 & 48 & 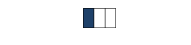 & 84 & 80 & 17 & 24 \\
\hline$\sigma$ & 22,8 & 25,5 & 11,9 & 43,5 & & 35,8 & 29,1 & 5,7 & 15,6 \\
\hline
\end{tabular}

Nota. AI refere-se às avaliações iniciais e AF, às finais para os grupos Equivalência (GEQ) e Equivalência mais Treino de Composição (GEQTC). 
foram expressas de forma absoluta, a diferença observada foi significativa $(p=0,01)$. Em relação aos grupos GEQTC e seus controles, as diferenças observadas entre as médias dos grupos não foram significativas tanto para frações absolutas $(p=0,5)$ quanto relativas $(p=0,14)$.

A análise dos desempenhos individuais mostrou que, no caso de frações absolutas, todos os participantes dos grupos experimentais apresentaram aumentos na porcentagem de acerto. No caso de frações relativas, entretanto, houve maior variabilidade entre os participantes. Três dos cinco participantes do grupo GEQ (P3, P4 e P5) e três do grupo GEQTC (P7, P8 e P9) mostraram aumentos nas porcentagens de acertos. Por outro lado, dois participantes do grupo GEQ (P1 e P2) apresentaram reduções na porcentagem de acerto e dois do grupo GEQTC (P6 e P10) não apresentaram mudanças no desempenho nas duas avaliações. Entre os participantes dos grupos de controle, no caso de frações absolutas, quatro participantes do grupo controle-GEQ (P12, P13, P14 e P15) e três do grupo controle-GEQTC (P16, P17 e P18) mostraram reduções nas porcentagens de acerto, enquanto os demais não mostraram diferenças entre as avaliações. No caso de frações relativas, os participantes P13 (grupo controle-GEQ) e P18 e P20 (grupo controle-GEQTC) mostraram diminuições, enquanto os demais apresentaram aumentos nas porcentagens de acertos na avaliação final.

A Tabela 4 apresenta as porcentagens (individuais e médias) de acerto nos pré- e pós-testes das condições $\mathrm{AB}, \mathrm{BC}$ e $\mathrm{AD}$ e o número de blocos de tentativas de treino necessários para os participantes dos grupos experimentais atingirem o critério na condição $\mathrm{AB} / \mathrm{BC}$.

As maiores porcentagens médias dos pré-testes ocorreram para o grupo GEQ, do que para o grupo GEQTC, em especial no pré-teste da condição AB (98\%). O teste $\mathrm{t}$ mostrou diferença significativa entre as porcentagens médias dos grupos experimentais nos pré-testes $\mathrm{AB}(p=0,03)$ e
$\mathrm{AD}(p=0,03)$, mas não no pré-teste $\mathrm{BC}(p=0,23)$. Quatro participantes do grupo GEQ (P1, P2, P3 e P4) e dois do grupo GEQTC (P7 e P9) tiveram 100\% de acertos nessa condição. $\mathrm{Na}$ condição BC, o mesmo ocorreu para P1 e P5 (GEQ) e P9 (GEQTC), e na condição AD, para P1, P3 e P5 (GEQ) e P6 (GEQTC). Todos os demais fizeram o treino direto dessas relações e tiveram $100 \%$ de acerto no pós-teste, sendo que o número de blocos de tentativas de treino foi menor na condição $\mathrm{AB}$ e $\mathrm{AD}$ e maior na condição $\mathrm{BC}$ (dados não mostrados na Tabela 4). No treino AB/BC, os participantes precisaram, em média, de aproximadamente dois blocos de tentativas para alcançarem o critério, não havendo diferenças entre os grupos

A Tabela 5 apresenta as porcentagens individuais de acerto por bloco de tentativas realizadas nos testes das condições $\mathrm{BA} / \mathrm{CB}$ (simetria), AC/CA (transitividade e equivalência) e expansão das classes $\{\mathrm{D}-(\mathrm{ABC})\}$, para os participantes dos grupos GEQ e GEQTC.

Os participantes de ambos os grupos experimentais atingiram o critério nos testes em um número comparável de blocos de tentativas. Todos os participantes do grupo GEQ e quatro do grupo GEQTC (P6, P7, P8 e P9) atingiram o critério no teste de simetria em no máximo três blocos de tentativas. Apenas P10 precisou de treino direto nessas relações. No teste de transitividade e equivalência, quatro participantes do grupo GEQ (P1, P3, P4 e P5) e quatro do GEQTC (P6, P7, $\mathrm{P} 8$ e P9) atingiram o critério em no máximo quatro blocos de tentativas. Apenas P2 (GEQ) e P10 (GEQTC) fizeram dois blocos de tentativas. No teste de expansão D-(ABC), todos os participantes dos grupos GEQ e GEQTC apresentaram decréscimo na porcentagem de acertos no último bloco de tentativas quando comparado ao bloco imediatamente anterior. As porcentagens médias de acerto no último bloco do grupo GEQ oscilaram entre $33 \%$ e $80 \%$, e do grupo GEQTC, entre $27 \%$ e $80 \%$.

Tabela 4. Porcentagem (individual e média) de acertos nos pré- e pós-testes dos treinos $A B, B C$, AD e número de blocos (N) realizados na condição $A B / B C$

\begin{tabular}{|c|c|c|c|c|c|c|c|c|}
\hline \multirow[b]{2}{*}{ Grupo } & \multirow[b]{2}{*}{ Part. } & \multicolumn{2}{|c|}{$\mathbf{A B}$} & \multicolumn{2}{|c|}{ BC } & \multirow{2}{*}{$\frac{\mathrm{AB} / \mathrm{BC}}{\mathrm{N}}$} & \multicolumn{2}{|c|}{ AD } \\
\hline & & Pré-teste & Pós-teste & Pré-teste & Pós-teste & & Pré-teste & Pós-teste \\
\hline & $\mathrm{P} 1$ & 100 & - & 100 & - & 2 & 100 & - \\
\hline & $\mathrm{P} 2$ & & & & & & & \\
\hline 100 & - & 78 & 100 & 4 & 78 & 100 & & \\
\hline \multirow[t]{6}{*}{ GEQ } & P3 & 100 & - & 33 & 100 & 2 & 100 & - \\
\hline & P4 & 100 & - & 55 & 100 & 2 & 67 & 100 \\
\hline & P5 & 89 & 100 & 100 & - & 2 & 100 & - \\
\hline & पा & 98 & - & 73 & 100 & 2.4 & 89 & 100 \\
\hline & P6 & 44 & 100 & 89 & 100 & 2 & 100 & - \\
\hline & P7 & 100 & - & 33 & 100 & 3 & 33 & 100 \\
\hline \multirow[t]{3}{*}{ GEQTC } & P8 & 33 & 100 & 33 & 100 & 2 & 56 & 100 \\
\hline & P9 & 100 & - & 100 & - & 2 & 67 & 100 \\
\hline & $\mathbf{\square}$ & 62 & 100 & 58 & 100 & 2.2 & 56 & 100 \\
\hline
\end{tabular}

Nota. GEQ refere-se ao grupo Equivalência e GEQTC, ao grupo Equivalência mais Treino de Composição. O sinal "-“ significa que o participante não fez a condição de pós-teste. 
A Tabela 6 apresenta as porcentagens (individuais e médias) de acerto nos teste de generalização 1 (TG1) e 2 (TG2) para os participantes dos grupos GEQ e GQTC. Todos os participantes mostraram porcentagens maiores no TG1 (médias iguais a $85 \%$ e $91 \%$ para GEQ e GEQTC, respectivamente) do que no TG2 (médias iguais a 50\% e 49,2\% para GEQ e GEQTC, respectivamente). Os participantes P1 e P5 (GEQ) e P6, P7 e P9 (GEQTC) tiveram 100\% de acertos no TG1, mas no TG2 nenhum participante apresentou esse valor. $\mathrm{O}$ teste $\mathrm{t}$ mostrou diferenças significativas apenas entre as porcentagens médias de acerto do TG1 e do TG2 para os grupos GEQ e GEQTC ( $p=0,03$ e 0,01 , respectivamente). A análise do desvio padrão mostrou menor variabilidade no TG1 para ambos os grupos ( $\sigma=17$ para GEQ e $\sigma=14$ para o GEQTC). No TG2, os valores foram $\sigma=31$ para o GEQ e $\sigma=21$ para o GEQTC.

A Tabela 7 apresenta a porcentagem de acertos e de erros ocorridos em cada relação testada na condição de teste de expansão da rede para os grupos GEQ e GEQTC. Em todas as relações, as porcentagens de acerto ficaram abaixo de 100\%. Em sete relações do GEQ (D1A1, B1D1, B2D2, B3D3, D1B1, D3B3 e C3D3) e em oito do GEQTC (D1A1, D2A2, B1D1, B2D2, B3D3, D1B1, D2B2 e D3B3), as porcentagens de acerto foram acima de $70 \%$. As menores porcentagens ocorreram em três relações do GEQ (C1D1, D2C2 e D3C3) e três do GEQTC (C1D1, C2D2 e D2C2), com valores máximos de $20 \%$ e $29 \%$, respectivamente.

A análise dos erros mostra que as respostas incorretas corresponderam mais frequentemente à escolha da comparação com o menor valor numérico no denominador (áreas sombreadas na Tabela 7). No grupo GEQ, esse fato ocorreu em 10 relações (D2A2, B1D1, B2D2, B3D3, D1B1, D3B3, C1D1, C3D3, D1C1 e D2C2) e no GEQTC, em oito (D2A2, B2D2, B3D3, D1B1, D3B3, C1D1, C3D3 e $\mathrm{D} 2 \mathrm{C} 2$ ). No grupo GEQ, a maior frequência de erros ocorreu pela escolha exclusiva da comparação com o menor valor numérico em quatro relações (B2D2, B3D3, D1B1 e D3B3) e em outras seis (D2A2, B1D1, C1D1, C3D3, D1C1, D2C2) os erros foram distribuídos entre as duas comparações

Tabela 5. Porcentagem individual de acerto por bloco de tentativas e nos testes das condições simetria BA/CB, transitividade e equivalência $(A C / C A)$ e expansão das classes $\{D-(A B C)\}$

\begin{tabular}{|c|c|c|c|c|c|c|c|c|c|c|c|c|c|}
\hline \multirow[b]{3}{*}{ Grupo } & \multirow[b]{3}{*}{ Part. } & \multirow{2}{*}{\multicolumn{3}{|c|}{$\begin{array}{l}\text { Simetria } \\
\text { Blocos }\end{array}$}} & \multirow{2}{*}{\multicolumn{4}{|c|}{$\begin{array}{c}\text { Transitividade/equivalência } \\
\text { Blocos }\end{array}$}} & \multirow{2}{*}{\multicolumn{5}{|c|}{$\begin{array}{c}\text { D-(ABC) } \\
\text { Blocos }\end{array}$}} \\
\hline & & & & & & & & & & & & & \\
\hline & & 1 & 2 & 3 & 1 & 2 & 3 & 4 & 1 & 2 & 3 & 4 & 5 \\
\hline \multirow{5}{*}{ GEQ } & $\mathrm{P} 1$ & 100 & 100 & - & 89 & 100 & - & - & 60 & 67 & 67 & 60 & - \\
\hline & $\mathrm{P} 2$ & 100 & 100 & - & 79 & 87 & 79 & - & 67 & 60 & - & - & - \\
\hline & P3 & 100 & 98 & 100 & 67 & 78 & 78 & 100 & 67 & 80 & 87 & 90 & 80 \\
\hline & P4 & 100 & 100 & - & 78 & 100 & - & - & 46 & 33 & - & - & - \\
\hline & P5 & 100 & 100 & - & 100 & 100 & - & - & 73 & 73 & 80 & 80 & 60 \\
\hline \multirow{5}{*}{ GEQTC } & P6 & 100 & 100 & - & 89 & 100 & - & - & 80 & 73 & - & - & - \\
\hline & P7 & 100 & 100 & - & 100 & 100 & - & - & 60 & 60 & 67 & 47 & - \\
\hline & P8 & 100 & 100 & - & 89 & 100 & - & - & 47 & 40 & - & - & - \\
\hline & P9 & 100 & 100 & - & 78 & 89 & 100 & - & 80 & 80 & 87 & 80 & - \\
\hline & P10 & 67 & 78 & 78 & 44 & 78 & 44 & - & 53 & 27 & - & - & - \\
\hline
\end{tabular}

Nota. GEQ refere-se ao grupo Equivalência e GEQTC, ao grupo Equivalência mais Treino de Composição. O sinal “-“ significa que o participante não fez o bloco.

Tabela 6. Porcentagem (individual e média) de acertos nos testes de generalização

\begin{tabular}{|c|c|c|c|c|c|c|c|}
\hline \multicolumn{8}{|c|}{ Condições } \\
\hline Grupo & Partic. & TG1 & TG2 & Grupo & Partic. & TG1 & TG2 \\
\hline \multirow{7}{*}{ GEQ } & $\mathrm{P} 1$ & 100 & 21 & & P6 & 100 & 54 \\
\hline & P2 & 92 & 80 & & P7 & 100 & 67 \\
\hline & P3 & 67 & 33 & GEQTC & P8 & 88 & 29 \\
\hline & $\mathrm{P} 4$ & 67 & 30 & & P9 & 100 & 71 \\
\hline & P5 & 100 & 87 & & P10 & 67 & 25 \\
\hline & -1 & 85 & 50 & & $\Pi$ & 91 & 49 \\
\hline & $\sigma$ & 17 & 31 & & $\sigma$ & 14 & 21 \\
\hline
\end{tabular}

Nota. TG1 refere-se ao teste de generalização 1 e TG2, ao teste de generalização 2 para os grupos Equivalência (GEQ) e Equivalência mais Treino de Composição (GEQTC). 
incorretas, mas continuaram mais frequentes nas frações com os menores denominadores. No grupo GEQTC, a escolha exclusiva pela comparação com o menor valor numérico no denominador da fração ocorreu em três relações (B3D3, D1B1 e C1D1) e em outras cinco (D2A2, B2D2, D3B3, C3D3 e D2C2), os erros foram distribuídos entre as duas comparações incorretas, mas com maior frequência naquelas com os menores valores nos denominares.

\section{Discussão}

Este experimento objetivou investigar o efeito do treino de relações condicionais entre estímulos fracionários na forma de figuras e numéricos e estímulos numéricos e numéricos, com e sem treino de composição sobre: (1) a formação de classes de equivalência com estímulos fracionários; (2) a expansão das classes de equivalência formadas e a generalização para novas situações; e (3) a resolução de problemas fracionários do tipo lápis e papel. Quanto ao primeiro objetivo, os resultados mostraram formação de classes de equivalência de estímulos tanto no grupo GEQ quanto GEQTC (Tabela 5); com relação ao segundo objetivo, as porcentagens de acerto observadas ao longo dos blocos do teste de expansão das classes foram menores para os dois grupos experimentais no último (Tabela 5). Nos testes de generalização, as porcentagens de acerto foram maiores no TG1 em relação ao TG2 para ambos os

Tabela 7. Porcentagem de acertos e erros em cada relação testadas na condição de teste de expansão da rede D-(ABC)

\begin{tabular}{|c|c|c|c|c|c|c|}
\hline \multirow{4}{*}{$\frac{\text { Relação }}{\text { D1A1 }}$} & \multicolumn{4}{|c|}{ GEQ } & \multicolumn{2}{|c|}{ GEQTC } \\
\hline & \multicolumn{4}{|c|}{ Erros } & \multirow{2}{*}{\multicolumn{2}{|c|}{$\begin{array}{c}\text { Erros } \\
\text { Alternativas }\end{array}$}} \\
\hline & A & \multicolumn{2}{|c|}{ Alternativas } & \multirow[t]{2}{*}{ A } & & \\
\hline & & $\mathrm{A} 2(1 / 4 \mathrm{~F})$ & $\mathrm{A} 3(1 / 5 \mathrm{~F})$ & & $\mathrm{A} 2(1 / 4 \mathrm{~F})$ & $\mathrm{A} 3(1 / 5 \mathrm{~F})$ \\
\hline$(3 / 9-1 / 3 F)$ & 96 & 0 & 4 & 82 & 0 & 18 \\
\hline D2A2 & & $\mathrm{A} 1(1 / 3 \mathrm{~F})$ & $\mathrm{A} 3(1 / 5 \mathrm{~F})$ & & $\mathrm{A} 1(1 / 3 \mathrm{~F})$ & $\mathrm{A} 3(1 / 5 \mathrm{~F})$ \\
\hline$(3 / 12-1 / 4 \mathrm{~F})$ & 50 & 37 & 12 & 79 & 14 & 7 \\
\hline D3A3 & & $\mathrm{A} 1(1 / 3 \mathrm{~F})$ & $\mathrm{A} 2(1 / 4 \mathrm{~F})$ & & $\mathrm{A} 1(1 / 3 \mathrm{~F})$ & $\mathrm{A} 2(1 / 4 \mathrm{~F})$ \\
\hline$(3 / 15-1 / 5 \mathrm{~F})$ & - & - & - & - & - & - \\
\hline B1D1 & & $\mathrm{D} 2(3 / 12)$ & D3(3/15) & & $\mathrm{D} 2(3 / 12)$ & D3(3/15) \\
\hline$(1 / 3-3 / 9)$ & 88 & 8 & 4 & 86 & 0 & 14 \\
\hline B2D2 & & $\mathrm{D} 1(3 / 9)$ & D3(3/15) & & D1(3/9) & D3(3/15) \\
\hline$(1 / 4-3 / 12)$ & 75 & 25 & 0 & 71 & 21 & 7 \\
\hline B3D3 & & D1(3/9) & $\mathrm{D} 2(3 / 12)$ & & D1(3/9) & D2(3/12) \\
\hline$(1 / 5-3 / 15)$ & 96 & 4 & 0 & 86 & 14 & 0 \\
\hline D1B1 & & $\mathrm{B} 2(1 / 4)$ & $\mathrm{B} 3(1 / 5)$ & & $\mathrm{B} 2(1 / 4)$ & $\mathrm{B} 3(1 / 5)$ \\
\hline$(3 / 9-1 / 3)$ & 87 & 12 & 0 & 79 & 21 & 0 \\
\hline D2B2 & & $\mathrm{B} 1(1 / 3)$ & B3(1/5) & & B1(1/3) & B3(1/5) \\
\hline$(3 / 12-1 / 4)$ & 67 & 16 & 16 & 86 & 7 & 7 \\
\hline D3B3 & & B1(1/3) & B2(1/4) & & B1(1/3) & B2(1/4) \\
\hline$(3 / 15-1 / 5)$ & 92 & 8 & 0 & 71 & 21 & 7 \\
\hline C1D1 & & $\mathrm{D} 2(3 / 12)$ & D3(3/15) & & $\mathrm{D} 2(3 / 12)$ & D3(3/15) \\
\hline$(2 / 6-3 / 9)$ & 25 & 62 & 12 & 14 & 86 & 0 \\
\hline C2D2 & & D1(3/9) & D3(3/15) & & D1(3/9) & D3(3/15) \\
\hline$(2 / 8-3 / 12)$ & 63 & 4 & 33 & 29 & 7 & 64 \\
\hline C3D3 & & $\mathrm{D} 1(3 / 9)$ & $\mathrm{D} 2(3 / 12)$ & & D1(3/9) & $\mathrm{D} 2(3 / 12)$ \\
\hline$(2 / 10-3 / 15)$ & 71 & 25 & 4 & 64 & 29 & 7 \\
\hline $\mathrm{D} 1 \mathrm{C} 1$ & & $\mathrm{C} 2(2 / 8)$ & $\mathrm{C} 3(2 / 10)$ & & $\mathrm{C} 2(2 / 8)$ & $\mathrm{C} 3(2 / 10)$ \\
\hline$(3 / 9-2 / 6)$ & 46 & 46 & 8 & 57 & 14 & 28 \\
\hline $\mathrm{D} 2 \mathrm{C} 2$ & & $\mathrm{C} 1(2 / 6)$ & $\mathrm{C} 3(2 / 10)$ & & $\mathrm{C} 1(2 / 6)$ & $\mathrm{C} 3(2 / 10)$ \\
\hline$(3 / 12-2 / 8)$ & 21 & 58 & 21 & 7 & 79 & 14 \\
\hline D3C3 & & $\mathrm{C} 1(2 / 6)$ & $\mathrm{C} 3(2 / 10)$ & & $\mathrm{C} 1(2 / 6)$ & $\mathrm{C} 2(2 / 8)$ \\
\hline$(3 / 15-2 / 10)$ & 29 & 33 & 38 & 57 & 14 & 28 \\
\hline
\end{tabular}

Nota. $\mathrm{A}=$ acertos. 
grupos experimentais (Tabela 6); e, por último, com relação ao terceiro objetivo, os dados mostraram variabilidade na porcentagem de acerto entre os participantes do grupo GEQ e GEQTC nos problemas expressos de forma relativa quando os desempenhos da avaliação inicial foram comparados aos da avaliação final (teste lápis e papel). A análise dos resultados mostrou haver diferença significativa entre as porcentagens médias de acerto apenas na avaliação final com frações absolutas para o grupo GEQ (Tabela 3).

Com relação à formação das classes de equivalência, os resultados dos testes $\mathrm{BA} / \mathrm{CB}$ (simetria) e $\mathrm{AC} / \mathrm{CA}$ (transitividade/equivalência) indicam a possibilidade de aprendizagem de novas relações a partir de outros treinos condicionais ( $\mathrm{AB}$ e $\mathrm{BC}$ ) também com estímulos fracionários, conforme sugerido por outros estudos (e.g., Sidman \& Tailby, 1982).

Conforme a literatura, classes de equivalência podem ser expandidas. Sidman, Kirk, e Willson-Morris (1985), por exemplo, demonstraram a formação de classes de equivalência de seis membros e consideraram as relações de transitividade e equivalência e de simetria como uma condição necessária para a ocorrência da expansão. Apesar da formação de classes de três membros com estímulos fracionários, os dados do presente estudo indicaram que os participantes do GEQ e do GEQTC não mantiveram as porcentagens de acerto ao longo dos blocos de tentativas na condição de teste de expansão das classes, como observado no estudo de Sidman et al. (1985). Essa inconsistência nos resultados pode ter sido ocasionada pelo tipo de estímulo utilizado (Sidman et al., 1985 usou letras gregas e o presente estudo, frações), como pode indicar que a ocorrência de relações de simetria e transitividade podem não ser condições suficientes para formação de classes com estímulos fracionários.

O melhor desempenho observado no TG1 em relação ao TG2 pode ser explicado com base na possibilidade dos participantes terem aplicado o conceito referencial de metade para resolver os problemas (Spinillo, 1992). Em outras palavras, no TG1, todos os exercícios poderiam ser resolvidos diretamente pela multiplicação ou divisão das frações por um mesmo número, o que não era possível no TG2, cuja solução dos problemas exigia a emissão de uma cadeia de respostas maior. Por exemplo, dividir os numeradores e os denominadores das frações por números diferentes, para em seguida comparar os resultados dessas operações e verificar se eles foram iguais (Spinillo,1993). De forma semelhante, no teste de expansão, a maior frequência de erros pela escolha da alternativa incorreta, em que estava a fração com o menor valor numérico no denominador, indica que a resposta não estava sob controle da relação existente entre as frações, mas apenas sob o controle das unidades menores que as compunham.

As menores porcentagens de acerto observadas na avaliação inicial, quando comparada com a avaliação final, e as maiores porcentagens de acerto observadas no pré-teste $\mathrm{AB}$ e menores nos testes $\mathrm{BC}$ e AD, para os grupos GEQ e GEQTC, corroboram dados da literatura sobre a dificuldade que alunos de início do sexto ano apresentam para resolver problemas fracionários com frações expressas de forma relativa (Carraher \& Schliemann, 1992; Santos, 1999; Santos, Cameschi, \& Hanna, 2009).

$\mathrm{Na}$ avaliação final para problemas com frações relativas, a variabilidade na porcentagem de acerto observada entre os participantes que fizeram treino de composição (GEQTC) e os que não fizeram (GEQ), indica a possibilidade de efeito de outras variáveis não manipuladas no presente experimento. Por outro lado, como houve aumento na porcentagem de acerto na avaliação final, quando comparada com a inicial, tanto dos participantes P3, P4 e P5 (GEQ) quanto dos participantes P7, P8 e P9 (GEQTC), tornam-se necessárias investigações adicionais sobre o possível efeito que o treino de composição pode ter sobre a resolução de problemas fracionários. Uma possibilidade é que a quantidade de treino de composição da resposta, programada no presente estudo, não viabilizou o desenvolvimento de controle pelas unidades menores que formavam as frações, as quais são estímulos necessários para identificar as figuras ou representações numéricas relacionadas a cada objeto composto. Por exemplo, a quantidade de treino para compor a fração 2/6 não produziu (1) o controle da resposta por uma parte do objeto apresentada na cor azul (no caso, dois toquinhos azuis) e o estabelecimento da relação entre essa parte do objeto e o numerador da fração numérica, e também (2) o controle por todos os toquinhos juntos (no caso, os dois azuis e mais os quatro brancos) e o estabelecimento da relação entre eles e o denominador da fração. Porém, como a literatura aponta que crianças com história de dificuldade em leitura e escrita apresentam melhora nessas habilidades após passarem por treino de composição de palavras a partir de unidades mínimas (de Rose et al., 1996; de Souza et al., 2009; Hübner et al., 2009), é possível supor que esses efeitos possam também ocorrer na aprendizagem de frações, uma vez que suas unidades mínimas são as partes em que o inteiro é dividido.

Diferenças entre os estímulos envolvidos no treino de composição para a aquisição de respostas de leitura e escrita e de conceitos fracionários pode explicar a semelhança nos resultados entre o GEQ e o GEQTC. No treino para a aquisição da escrita pode haver correspondência formal e funcional, com predominância para a formal, entre as unidades de controle e os produtos das respostas. $\mathrm{Na}$ composição das frações, a correspondência é essencialmente funcional, visto haver poucas semelhanças físicas entre os estímulos, o que pode tornar a aprendizagem de conceitos matemáticos mais difícil do que a de leitura. Por exemplo, há pouca correspondência formal entre um retângulo dividido em três partes iguais, sendo duas brancas e uma azul, e a fração $1 / 3$ ou 2/6. Também não há semelhança formal, ou se há, ela é mínima, entre as frações $1 / 3$ e $2 / 6$. Portanto, essas relações matemáticas são basicamente simbólicas, o que pode dificultar a aprendizagem do conceito de proporção apenas pelo treino de composição, uma vez que esse conceito também é abstrato. Assim, é possível que a aprendizagem de frações necessite de controle discriminativo adicional, como aquele proporcionado pelo uso de regras. Seria interessante que, em estudos posteriores, fosse avaliado se a contribuição do treino de composição para a aquisição do conceito de proporção seria aumentada caso regras sobre a resolução de problemas fracionários fossem utilizadas. 
Concluindo, os resultados mostraram a formação de classes de equivalência com estímulos fracionários na forma de figuras e numéricos, níveis discretos de expansão dessas classes e a possibilidade de generalização da resposta para novas situações. Contudo, não foram obtidas evidências de que o treino de composição pode facilita a aprendizagem do conceito de proporção.

\section{Referências}

Albuquerque, R. A., \& Melo, R. M. (2005). Equivalência de estímulos: conceito, implicações e possibilidades de aplicação. In J. Abreu-Rodrigues, \& M. R. Ribeiro (Eds.), Análise do comportamento: pesquisa, teoria e aplicação (pp. 245-264). Porto Alegre: ArtMed.

Carmo, J. S. (2000). O conceito de número como rede de relações. In R. R. Kerbauy (Ed.), Sobre comportamento e cognição: conceitos, pesquisa, aplicação, a ênfase no ensinar, na emoção e no questionamento clínico (Vol. 5, pp. 97-113). Santo André: SET.

Carraher, T. N., Carraher, D. W., \& Schlieman, A. D. (1986). Proporcionalidade na educação científica e matemática: desenvolvimento cognitivo e aprendizagem. Revista Brasileira de Estudos Pedagógicos, 67, 586-602.

Carraher, D. W., \& Schliemann, A. D. (1992). A compreensão de frações como magnitudes relativas. Psicologia: Teoria e Pesquisa, 8(1), 67-78.

de Rose, J. C., Souza, D. G., Rossito, A. L., \& de Rose, T. M. S. (1989). Aquisição de leitura após história de fracasso escolar: equivalência de estímulos e generalização. Psicologia: Teoria e Pesquisa, 5(3), 325-346.

de Rose, J. C., de Souza, D. G., \& Hanna, E. S. (1996). Teaching reading and spelling: Exclusion and stimulus equivalence. Journal of Applied Behavior Analysis, 29, 451-469.

de Rose, J. C. (2005). Analise comportamental da aprendizagem de leitura e escrita. Revista Brasileira de Análise do Comportamento, 1, 29-50.

de Rose, J. C. (2010). Prefácio. In J. S. Carmo, \& P. S. T. Prado, (Eds.), Relações simbólicas e aprendizagem da matemática (pp 49-68). Santo André: ESETec.

de Souza, D. G., de Rose, J. C., Faleiros, T. C., Bortoloti, R., Hanna, E. S., \& McIlvane, W. J. (2009). Teaching generative reading via recombination of minimal textual units: A legacy of verbal behavior to children in Brazil. Revista Internacional de Psicología y Terapia Psicológica, 9, 19-44.

Green, G. (2010). A tecnologia de controle de estímulos no ensino de equivalências número quantidade. In J. S. Carmo, \& P. S. T. Prado (Eds.), Relações simbólicas e aprendizagem da matemática (pp 7 -12). Santo André: ESETec.
Hübner, M. M. C., Gomes, R. C., \& McIlvane, W. (2009). Recombinative generalization in minimal verbal unit-based reading instruction for pre-reading children. Experimental Analysis of Human Behavior Bulletin, 27, 11-17.

Lynch, D. C., \& Cuvo, A. J. (1995). Stimulus equivalence instruction of fraction-decimal relations. Journal of Applied Behavior Analysis, 28, 115-126.

Ninness, C., Dixon, M., Barnes-Holmes, D., Rumph, R., McCuller, G., Ford, A. M., \& Elliott, M. P. (2006). Transformation of mathematical and stimulus functions. Journal of Applied Behavior Analysis, 39, 299-321.

Ninness, C., Dixon, M., Barnes-Holmes, D., Ruhfelot, R. A., Rumph, R., McCuller, G., \& McGinty, J. (2009). Constructing and deriving reciprocal trigonometrics relations: A functional analysis approach. Journal of Applied Behavior Analysis, 42, 191-208.

Santos, A. C. G., Souza, A. V., \& Bay, C. K. (1997). Aprendizagem do conceito de proporção: interação entre equivalência de estímulos e instruções. [Resumo]. In Sociedade Brasileira de Psicologia (Ed.), Anais da XXVII Reunião Anual da Sociedade Brasileira de Psicologia. Campinas: Sociedade Brasileira de Psicologia.

Santos, A. C. G. (1999). Equivalência de frações: como anda a compreensão dos alunos? Estudos: Revista da Universidade Católica de Goiás, 26, 233-249.

Santos, A. C. G., Cameschi, C., \& Hanna, E. S. (2009). Ensino de frações baseado no paradigma de equivalência de estímulos. Revista Brasileira de Análise do Comportamento, 5, 19-41.

Sidman, M. (1971). Equivalence relations and behavior: A research story. Boston: Author Cooperative.

Sidman, M., \& Cresson, O. (1973). Reading and transfer of crosmodal stimulus equivalences in severe retardation. Equivalence relations and behavior: a research story. Author Cooperative, Inc: Boston, Massachusets.

Sidman, M., \& Tailby, W. (1982). Conditional discrimination vs. matching to sample: an expansion of the testing paradigm. Journal of the Experimental Analysis of Behavior, 37, 5-22.

Sidman, M., Kirk, B., \& Willson-Morris, M. (1985). Six-member stimulus classes generated by conditional-discrimination procedure. Journal of the Experimental Analysis of Behavior, $37,5-22$.

Skinner, B. F. (1978). O comportamento verbal. São Paulo: Cultrix.

Spinillo, A. G. (1992). A importância do referencial de metade e o desenvolvimento do conceito de proporção. Psicologia: Teoria e Pesquisa, 8(3), 305-317.

Spinillo, A. G. (1993). As relações de primeira ordem em tarefas de proporção: uma outra explicação quanto às dificuldades das crianças. Psicologia: Teoria e Pesquisa, 9(2), 349-364. 